\title{
Aplicação do time-driven ABC em uma empresa varejista
}

\author{
Antônio Artur de Souza \\ Doutorado em Management Science pela The University of Lancaster \\ Professor Associado na Universidade Federal de Minas Gerais - UFMG \\ Avenida Antônio Carlos, 6627. Pampulha. Belo Horizonte/MG. CEP: 31270-901 \\ E-mail: artur@face.ufmg.br
}

Ewerton Alex Avelar

Mestrado em Administração pela Universidade Federal de Lavras - UFLA Pesquisador do Núcleo de Estudos Gerenciais e Contábeis da Universidade Federal de Minas Gerais - UFMG Avenida Antônio Carlos, 6627. Pampulha. Belo Horizonte/MG. CEP: 31270-901 E-mail: ewertonaavelar@gmail.com

Terence Machado Boina Especialização em Gestão Pública pela Fundação João Pinheiro -FJP Analista Administrativo na Agência Nacional do Cinema - ANCINE Avenida Graça Aranha, 35. Centro. Rio de Janeiro/RJ. CEP: 20030-002

E-mail:tmboina@yahoo.com.br

Neiva Andrade Caires Mestrado em Ciências Contábeis pela Universidade de Minas Gerais - UFMG Pesquisadora do Núcleo de Estudos Gerenciais e Contábeis da Universidade Federal de Minas Gerais - UFMG Avenida Antônio Carlos, 6627. Pampulha. Belo Horizonte/MG. CEP: 31270-901 E-mail: neiva.caires@hotmail.com

\section{RESUMO}

Este artigo apresenta os resultados de uma pesquisa exploratória, qualitativaquantitavia, que objetivou propor um modelo do time-driven activity-based costing (TDABC) para uma empresa varejista distribuidora de materiais elétricos, localizada em Belo Horizonte/MG. Foram utilizadas as seguintes técnicas para a coleta de dados: entrevistas semi-estruturadas, entrevistas não-estruturadas, análise documental e observação participante. A pesquisa foi realizada entre os anos de 2008 e 2009. Observou-se a necessidade do desenvolvimento de apenas algumas equações de tempo (time equations) para representar as principais atividades de cada departamento da empresa estudada. Salienta-se o fato de que algumas dessas atividades, bastante complexas, terem sido modeladas sem maiores problemas. Ressalta-se, também, a contribuição substancial das planilhas eletrônicas do Microsoft巴® Excel (MS-Excel) para o desenvolvimento do modelo TDABC proposto. Verificou-se que o TDABC realmente apresenta vantagens em relação ao $A B C$ convencional como a facilidade em modelar atividades complexas por meio das equações de tempo e o baixo tempo de desenvolvimento do modelo. Contudo, também foram identificadas algumas limitações 
da abordagem do TDABC no estudo realizado. Primeiramente, os pesquisadores constataram um grau de subjetivismo inerente à metodologia. Ademais, a falta de padronização de algumas atividades impediu sua modelagem por meio das equações de tempo. Estudos futuros poderiam replicar essa pesquisa em outras empresas, além de abranger outros setores, a fim de corroborar ou não os resultados apresentados neste trabalho.

Palavras-chave: Custeio Baseado em Atividades. Time-driven ABC. Tomada de decisão.

\section{Application of time-driven $A B C$ in a retailer company}

\section{ABSTRACT}

This paper presents the results of a qualitative and exploratory research that aimed at developing a time-driven activity-based costing (TDABC) model for a retailer company. This company sells electrical material and it is located in Belo Horizonte/MG. The researchers used three techniques for collecting data: semi-structure interviews and non-structured, documental analysis and direct observation. The research was developed between 2008 and 2009. It was verified the development of only few time equations for representing the main activities of each company's department. It is important to enhance the fact of some very complex activities could be modeled without problems, and the relevant contribution of electronic spread-sheet of Microsoft@ Excel (MS-Excel) to develop the purposed model. It was observed that the TDABC really presents some advantages over the conventional activity-based costing (ABC), such as the ease of modeling the time equations and the low time of development of the model. However, some limitations of TDABC also were identified. Firstly, the researchers verified a degree of subjectivism inherent to methodology. Further, the nonstandardization of some activities what blocked their modeling through the time equations. Future researches could develop the study in other companies and expand it for other industries in order to ratify or not the results presented in this paper.

Keywords: Activity-based costing. Time-driven ABC. Decision making.

\section{INTRODUÇÃO}

De maneira geral, os métodos de custeio fornecem informações de custos que podem ser usadas para subsidiar satisfatoriamente o processo decisório. Por meio dessas informações, os gestores usualmente conseguem conhecer diversos aspectos importantes para as empresas no que tange ao processo de tomada de decisão, tais 
como: a rentabilidade/ lucratividade de clientes/produtos/serviços, a análise dos processos produtivos e administrativos, a precificação dos objetos de custos, os orçamentos e a avaliação de desempenho (BLOCHER et al., 2002).

Devido a sua característica principal - relacionar o consumo de recursos com atividades e, posteriormente, alocar custos aos objetos de custos -, o método de custeio baseado em atividades (activity-based costing - ABC) se configura, de modo geral, como uma alternativa para aperfeiçoar o processo decisório nas empresas (BRIMSON, 1996; KAPLAN; COOPER, 1998; ATKINSON et al., 2008). Uma das principais vantagens desse método é a geração de informações precisas sobre os custos dos recursos consumidos na produção de bens e na prestação de serviços.

A despeito dessas vantagens, autores como Goldratt e Cox (1997), Corbett Neto (1997) e Roni e Geri (2005) criticam a confiabilidade das informações de custos geradas pelo referido método de custeio no que tange à tomada de decisão, principalmente em função dele, geralmente, não considerar os gargalos de produção e de considerar que a relação entre o consumo de recursos e o nível de atividades é linear. Além dessas limitações, segundo Varilla et al. (2007), o ABC tem dificuldades em modelar atividades complexas e variáveis que utilizam diferentes métodos de trabalho.

Com o objetivo de dirimir as limitações do ABC, Kaplan e Anderson (2004) propuseram outra abordagem para esta metodologia de custeio: o Time-driven $A B C$ (TDABC). Segundo esses autores, o TDABC é um método adequado para subsidiar o processo decisório em processos complexos e dinâmicos, como em hospitais, indústrias de produção sob encomenda e empresas varejistas (KAPLAN; ANDERSON, 2007).

Nesse contexto, este artigo apresenta os resultados de uma pesquisa exploratória e qualitativa que objetivou propor um modelo do TDABC para uma empresa varejista distribuidora de materiais elétricos, localizada na região metropolitana de Belo Horizonte. Essa pesquisa teve como objetivos específicos: (a) modelar as principais atividades da empresa estudada; (b) desenvolver as equações de tempo para as atividades da empresa; e (c) identificar benefícios e as limitações do TDABC na empresa varejista estudada, tendo em vista suas peculiaridades. 
Pode-se dizer que pesquisas desenvolvidas sobre o TDABC se tornam cada vez mais relevantes, uma vez que essa nova abordagem de metodologia de custeio está sendo empregada em diversas empresas que atuam em diferentes setores (SOUZA et al., 2010). Todavia, verificou-se no levantamento bibliográfico que em empresas varejistas de pequeno porte o TDABC ainda não foi muito aplicado até o momento. Assim sendo, o caráter exploratório desta pesquisa se torna mais pujante.

Este artigo está dividido em 6 seções, contando com esta introdução. Na seção 2 são destacados conceitos relacionados ao ABC (subseção 2.1) e ao TDABC (subseção 2.2). Na seção 3 , por sua vez, apresenta-se a metodologia utilizada na pesquisa. Os resultados são descritos e discutidos nas seções 4 e 5 . Na seção 6 apresentam-se as conclusões do trabalho.

\section{REFERENCIAL TEÓRICO}

\subsection{Custeio baseado em atividades (Activity-based Costing - ABC)}

De acordo com Kaplan e Cooper (1998), o ABC tem como característica basilar a alocação dos custos dos recursos às atividades, e destas aos objetos de custos/custeio (produtos, serviços, processos, clientes etc.). Essa alocação ocorre por meio da identificação, da classificação e da mensuração do consumo de recursos pelas atividades e, em seguida, do consumo das atividades pelos objetos de custos. Pode-se dizer que, considerando que são diversas as atividades de uma empresa, o ABC utiliza um maior número de bases de alocação com a finalidade de identificar, em termos monetários, as atividades que efetivamente consumem os recursos (RADELLI; DURAN, 2000).

$\mathrm{O} A B C$ utiliza direcionadores para rastrear e mensurar o consumo dos recursos e das atividades. A mensuração é feita, de maneira geral, a partir de critérios mais apurados do que aqueles utilizados nos métodos de custeio tradicionais, como o custeio por absorção (SILVA, 2003). Segundo Cooper e Kaplan (1991), os direcionadores de custos buscam refletir a parcela de demanda que incide sobre cada produto individualmente. Radaelli e Duran (2000) acrescentam que a utilização de 
direcionadores de custos específicos para cada atividade permite apurar com relativa precisão o volume de recursos consumidos pelos produtos e/ou serviços.

Pode-se dizer que, ao proporcionar uma alocação mais criteriosa dos custos indiretos a partir do conhecimento das atividades desenvolvidas pela empresa, o $A B C$ normalmente apresenta-se como um substituto aos métodos de custos tradicionais no que tange à tomada de decisão (GOULART, 2000). Na Figura 1, apresenta-se de forma simplificada a relação entre atividades, recursos e custos.

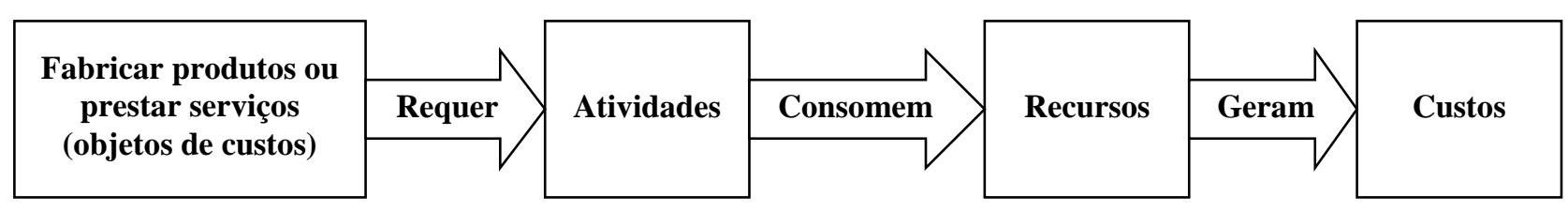

Figura 1: Relação entre atividades, recursos e custos sob a perspectiva do ABC Fonte: Elaborado pelos autores

Segundo Cooper e Kaplan (1991), sob a perspectiva do ABC, deve se segregar as atividades em quatro diferentes níveis: unidade, lote, produto e suporte às instalações. Hirsch (2000) destaca que as atividades classificadas no nível da unidade são referentes às necessárias para a elaboração de apenas uma unidade de um determinado produto (material direto, por exemplo). Por outro lado, as atividades classificadas no nível do lote são as demandadas cada vez que um lote do produto é fabricado (inspeção, por exemplo). Por sua vez, como atividades no nível do produto são classificadas aquelas necessárias para dar suporte ao desenvolvimento de determinado produto (projeto, por exemplo), enquanto, as atividades classificadas no nível das instalações são as que dão suporte à estrutura geral da empresa (depreciação da fábrica, por exemplo) (KAPLAN; COOPER, 1998).

Conforme Silva (2003), o ABC analisa a empresa de forma horizontal, ao dividi-la em processos, pois ao fracionar a empresa em atividades, o sistema $A B C$ usa um maior número de direcionadores, o que proporciona uma melhor distribuição dos custos fixos indiretos. De acordo com Malmi (1999), o ABC foi apresentado inicialmente como um método para alocar custos aos produtos das empresas. Todavia, tendo em vista sua 
repercussão na apuração monetária das atividades da empresa, o $A B C$ foi descrito como um método para analisar consumo de recursos da empresa e, conseqüentemente, como uma ferramenta de gestão.

Diversos aspectos positivos na utilização do método $A B C$ são ressaltados na literatura por autores como Kaplan e Cooper (1998), Hansen e Mowen (2006), Atkinson et al. (2008), e Majid e Sulaiman (2008): (i) alocação dos gastos indiretos aos objetos de custos, por meio de critérios mais apurados que os demais métodos; (ii) minimização/eliminação dos problemas de os produtos serem supercusteados ou subcusteados (como habitualmente acontecem nos demais métodos de custeio); (iii) progresso contínuo dos processos da entidade, a partir da melhora na apuração dos custos; (iv) aprimoramento da precificação dos objetos de custos, tendo em vista a obtenção de informações precisas dos custos da empresa; e (v) apuração aperfeiçoada das informações de rentabilidade/lucratividade por cliente/mercado.

Contudo, vários estudos como os conduzidos por Roni e Geri (2005), Kaplan e Anderson (2007), e Wegmann e Nozile (2008), ressaltam alguns fatores que restringem a utilização do $A B C$ nas empresas de uma forma geral: (a) custo elevado de implementação e manutenção; (b) fracassos na utilização do referido método, notadamente em função da diversidade e da complexidade das atividades; (c) desconsideração de aspectos relacionados aos gargalos; (d) alto grau de subjetividade na definição de direcionadores; e (e) lentidão excessiva na implementação do método e na geração de relatórios. No intuito de suprimir/atenuar as limitações do $A B C$ convencional, principalmente as referentes à morosidade $\mathrm{e}$ ao alto custo de implementação e manutenção da metodologia, Kaplan e Anderson (2004) apresentam uma possível solução: o TDABC.

\subsection{Time-driven Activity-based Costing (TDABC)}

O TDABC, nova abordagem do ABC, foi desenvolvido em 1997 por Steven Anderson e, posteriormente, implementada em algumas empresas. Em 2001, Anderson aperfeiçoou essa metodologia com o auxílio do professor Robert Kaplan da Harvard Business School (EVERAERT et al., 2008b). De acordo com Demeere et al. (2009), os 
proponentes do TDABC afirmam que essa nova metodologia demanda menos tempo de pesquisa e de implementação do que o $A B C$ convencional. Segundo Kaplan e Anderson (2004), o TDABC deve ser entendido como outra "opção" na gestão de custos das empresas.

A utilização do $A B C$ convencional é usualmente limitada quando há uma grande diversidade de recursos usados por variadas atividades. Nesses casos, as empresas geralmente têm dificuldade para identificar e para analisar informações de custos. Todavia, em complexos e dinâmicos ambientes empresariais, é essencial obter informações acuradas de custos para cada minuto das atividades desenvolvidas nas empresas (tal como propõe o TDABC), a fim de subsidiar satisfatoriamente o processo decisório (EVERAERT et al., 2008a).

A abordagem do TDABC requer a estimação de somente dois parâmetros: o custo unitário (normalmente medida em minuto) da capacidade prática (efetiva) e o número de unidades requeridas desta capacidade para desempenhar uma atividade ou uma tarefa desta atividade (BRYON et al., 2009). Sob a perspectiva do TDABC, a capacidade significa o "tempo utilizado pelos empregados" (por exemplo, em trabalhos manuais) ou "espaço demandado" (por exemplo, em atividades domésticas). O tempo necessário para desempenhar uma atividade é modelado com base em todas as variáveis relacionadas.

As características que direcionam as variáveis da atividade são chamadas de direcionadores de tempo (time-drivers), uma vez que "direcionam" o tempo despendido para um caso particular (KAPLAN; ANDERSON, 2007). As equações de tempo (time equations), um dos fundamentos do TDABC, determinam como os direcionadores de tempo orientam o tempo despendido para cada atividade. Em ambientes complexos, onde o tempo necessário para desempenhar uma atividade é orientado por muitos direcionadores, o TDABC possibilita a inclusão de múltiplos direcionadores de tempo para cada atividade (CARDINAELS; LABRO, 2008). Destaca-se que, sob a perspectiva do TDABC, os direcionadores de custos podem ser classificados em contínuos, discretos ou indicadores (BRUGGEMAN; ANDERSON; LEVANT, 2005). 
Conforme Everaert et al. (2008a), o processo de implementação do TDABC em uma empresa pode ser descrito em seis etapas: (1) identificação dos recursos utilizados pelas atividades, dividindo-os em grupos; (2) estimativa total dos custos de cada grupo de recursos; (3) mensuração da capacidade efetiva das atividades, ou seja, desconsideram-se tempo de treinamento e reuniões, por exemplo; (4) cálculo do custo unitário de cada grupo de recursos - divide-se o valor encontrado por grupo de recursos pela capacidade prática da atividade; (5) determinação do tempo requerido por evento de uma atividade, baseando-se na equação de tempo para cada atividade e nas características do evento; e (6) multiplicação do custo unitário (obtido na etapa 4), pelo tempo requerido por evento (etapa 5 ).

Diversos benefícios podem ser obtidos pelas empresas por meio do TDABC, segundo destacam Pernot et al. (2007): (i) rapidez e flexibilidade na construção de modelos; (ii) aplicabilidade, de forma mais simples e fácil, em complexas empresas, uma vez que utiliza variados direcionadores que refletem as diversas características especiais identificadas nas atividades; (iii) geração tempestiva de relatórios, possibilitando um feedback mais rápido aos gestores; (iv) facilidade de integração com os sistemas de gestão integrada (Enterprise Resource Planning - ERP) existentes nas empresas; (v) baixo custo de implementação e manutenção; e (vi) utilização em empresas com grande e complexa variedade de produtos, clientes, fornecedores, canais de distribuição.

Para demonstrar um exemplo de aplicação do TDABC, optou-se para apresentar um estudo de caso descrito e analisado por Everaert et al. (2008a). Esse estudo foi realizado na empresa Sanac Inc, uma distribuidora de produtos voltados para agricultura, casa e jardim. A Sanac tem aproximadamente 7.000 clientes, 7.000 produtos, emprega 129 pessoas e recebe aproximadamente 298.000 ordens de pedidos por ano.

Em junho de 2005, a Sanac começou a implementar o método ABC para apuração de seus custos, contudo, devido ao grande número de dados, ficou definido que o TDABC era o método mais apropriado para o ambiente onde as operações e os processos variam rapidamente. Uma das primeiras atividades selecionadas para a 
modelagem no TDABC foi a "entrega" dos produtos, ou seja, foi definida uma equação de tempo calculada para cada entrega. O tempo foi calculado, após entrevistas com os funcionários, conforme a seguinte equação apresentada no Quadro 1. O Quadro 2 apresenta a descrição das variáveis da equação.

Atividade

Entrega das mercadorias

Quadro 1: Equação de tempo formulada para a atividade de entrega de mercadorias da Sanac Inc.

Fonte: Adaptado de Everaert et al. (2008a)

\begin{tabular}{|c|c|}
\hline Atividade & Descrição da variável \\
\hline \multirow{4}{*}{ Entrega das mercadorias $(\mathrm{EM})$} & $\mathrm{X} 1 \rightarrow$ Número do pedido \\
\cline { 2 - 2 } & $\mathrm{X} 2 \rightarrow$ Número de unidades \\
\cline { 2 - 2 } & $\mathrm{X} 3 \rightarrow$ Número de pallets cheios \\
\cline { 2 - 2 } & $\mathrm{X} 4 \rightarrow$ Número de viagens \\
\cline { 2 - 2 } & $\mathrm{X} 5 \rightarrow$ Número de pallets embrulhados \\
\hline
\end{tabular}

Quadro 2: Descrição das variáveis da equação de tempo formulada para a atividade de entrega de mercadorias da Sanac Inc.

Fonte: Adaptado de Everaert et al. (2008a)

Os autores consideraram que o TDABC é mais adequado para ambientes complexos, dinâmicos, e altamente competitivos. No estudo, foram destacados os seguintes pontos: (i) o custo de implementação foi de 11 homens-dia, $60 \%$ dos quais estavam fora de empresa; (ii) menos de um ano depois, a companhia estava usando os relatórios TDABC mensais prosperamente; (iii) nos relatórios, foi demonstrado que a maioria dos clientes de varejo (casa e jardim) tinha a menor rentabilidade e que usavam o poder de negociação do passado para adquirir a preços baixos e receber serviços extremamente qualificados; (iv) foram reduzidas as entregas desses clientes por semana; (v) foram renegociados preços e entregas condicionadas.

\section{METODOLOGIA}

A pesquisa, cujos resultados são apresentados neste artigo, pode ser classificada como exploratória e qualitativa-quantitativa, consistindo de um estudo de 
caso desenvolvido em uma empresa varejista mineira, com início em meados do semestre de 2008. De acordo com Malhotra (2003), a pesquisa exploratória descritiva qualitativa-quantitativa proporciona melhor visão e compreensão do contexto do problema. Para Yin (2005, p. 23), o estudo de caso

é uma forma de se fazer pesquisa empírica que investiga fenômenos contemporâneos dentro de seu contexto de vida real, em situações em que as fronteiras entre o fenômeno e o contexto não são claramente estabelecidas, onde se utiliza múltiplas fontes de evidência.

Foram utilizadas para a coleta de dados as seguintes técnicas: entrevistas semiestruturadas, entrevistas não-estruturadas, análise documental e observação participante. A pesquisa de campo foi realizada de forma intensiva por um pesquisador, que realizou coleta de dados na empresa todos os dias por um período de 6 meses, numa jornada de 4 horas diárias. Assim, foram 130 dias de coleta de dados e de análises in loco dos resultados.

As entrevistas semi-estruturadas foram realizadas com os principais funcionários de cada departamento da empresa. Essas entrevistas visaram compreender as peculiaridades das atividades desempenhadas em cada departamento, tais como: as variáveis que as influenciam e os instrumentos utilizados para a sua consecução. As entrevistas não-estruturadas, por sua vez, foram realizadas para esclarecer alguns aspectos considerados dúbios pelos pesquisadores.

A análise documental, por outro lado, visou analisar os documentos próprios de cada departamento, procurando indícios de como uma atividade era desenvolvida e seus possíveis direcionadores de custos. Também foram analisados alguns documentos enviados pela empresa externa responsável pela Contabilidade da empresa estudada (em especial a Folha de Pagamento) e alguns relatórios gerados pelo ERP utilizado na empresa.

Por fim, no tocante à observação participante, os pesquisadores acompanharam e observaram a execução de diversas atividades dos funcionários relatados anteriormente. Nesse contexto, ocorreram algumas conversações importantes com 
determinados funcionários, o que permitiu a obtenção de algumas informações relevantes sobre os processos internos da Empresa Beta. Ressalta-se que, em alguns casos (especificamente no Departamento Financeiro), os pesquisadores tiveram a possibilidade de desempenhar a atividade. Isso propiciou uma melhor avaliação dos tempos e das variáveis que influenciam as atividades por parte dos pesquisadores.

Foi desenvolvida uma triangulação dos dados coletados por meio das outras técnicas de coleta de dados utilizadas. Como destaca Yin (2005), a triangulação dos dados corresponde ao processo de confrontação dos dados coletados por meio de diferentes técnicas de coleta de dados, validando, ou não, a confiabilidade dos mesmos.

\section{RESULTADOS DO ESTUDO EMPÍRICO}

\subsection{Estudo de caso e desenvolvimento do modelo}

Nesta seção apresenta-se o estudo de caso abordado na pesquisa empírica. Trata-se de uma empresa familiar que atua como distribuidora de materiais elétricos. No intuito de resguardar informações sigilosas sobre a empresa estudada, utiliza-se o pseudônimo "Empresa Beta". De acordo com a Lei Complementar n 123/06, essa empresa pode ser considerada como de pequeno porte, uma vez que aufere receita anual acima de $R \$ 240$ mil e abaixo de $R \$ 2,4$ milhões (BRASIL, 2006).

A Empresa Beta foi fundada na década de 1960 e atua no setor varejista, distribuindo materiais elétricos industriais. Basicamente, a empresa pode ser segregada em quatro departamentos principais: Financeiro, Vendas e Almoxarifado, subordinados à Gerência. Boa parte das atividades desempenhadas na empresa, dentre elas a Contabilidade e a Tecnologia da Informação, é terceirizada no intuito de manter sua estrutura enxuta. Apesar do pequeno porte, a Empresa Beta possui centenas de clientes e comercializa mais de 4.000 itens distintos.

Para o desenvolvimento do modelo, primeiramente, os pesquisadores levantaram as informações de custos referentes aos recursos existentes e utilizados em cada departamento da empresa. Esses recursos foram identificados in loco, por meio 
da observação participante e de entrevistas semi-estruturadas e não-estruturadas. Grande parte dos dados e informações de custos foi coletada a partir de duas fontes de evidência principais: folha de pagamento da empresa e relatórios do ERP. As informações de custos relacionados à mão-de-obra foram coletadas utilizando-se relatórios elaborados por uma empresa de contabilidade externa.

Os demais dados e informações de custos, contudo, foram obtidos por meio de relatórios emitidos pelo ERP da empresa. Esses dados de custos foram atualizados monetariamente por meio do índice de preço ao consumidor (IPCA) calculado pela Fundação Instituto de Pesquisas Econômicas, Administrativas e Contábeis de Minas Gerais (IPEAD/MG). Essa fundação apura o IPCA com base na inflação registrada na região metropolitana de Belo Horizonte (onde se localiza a empresa estudada). Os custos identificados em cada departamento, tais como a depreciação e o material de escritório, foram alocados a cada atividade. Os custos com aluguel, por sua vez, foram alocados com base nos metros quadrados ocupados por departamento. Os custos que não puderam ser identificados, de forma lógica, a um dado departamento foram excluídos do modelo, a fim de se eliminar possíveis vieses.

Os custos com os serviços gerais como: limpeza das áreas da empresa e produção de lanches destinados aos funcionários, foram alocados com base em um índice calculado no número de funcionários e na área ocupada por departamento. Ressalta-se que essa pode ser considerada uma atividade secundária, de acordo com Brimson (1996) e Boiverst (1999), e que esse tipo de atividade ainda não é tratado explicitamente na literatura disponível sobre o TDABC.

A atividade "montagem de painéis eletrônicos" não foi considerada no desenvolvimento do modelo, notadamente devido a sua natureza não-estruturada. Conforme constataram os pesquisadores, a atividade não apresenta um nível razoável de padronização, sendo este um dos pressupostos básicos preceituados por Kaplan e Anderson (2007) para a adequada aplicabilidade do TDABC. Essa constatação ratifica o exposto por Sherrat (2005) e Souza et al. (2010).

Posteriormente ao cálculo do custo de cada departamento, analisaram-se as atividades realizadas por eles. Para identificar, entender e modelar as atividades foram 
utilizadas, intensivamente, a observação e as entrevistas. As atividades foram modeladas por meio do MS-Excel.

Para calcular o custo por minuto de cada departamento (input essencial ao modelo TDABC), estimou-se a capacidade prática (efetiva) de cada departamento. Nesse sentido, optou-se pela orientação de Kaplan e Anderson (2004), que estipula a capacidade prática em aproximadamente $80 \%$ da capacidade teórica (disponível). Para se calcular essa capacidade efetiva, então, considerou-se o tempo mensal disponível de cada funcionário de cada um dos departamentos multiplicado por 0,8.

\subsection{Modelo proposto}

Com base na coleta de dados realizada no Almoxarifado, foram identificadas e modeladas as seguintes atividades: "Receber e estocar mercadorias" (RE) e "Identificar e expedir mercadorias" (IE). Na Tabela 1, são apresentados os custos apurados mensalmente no Almoxarifado; enquanto na Tabela 2 apresentam-se as equações de tempo definidas para as atividades desenvolvidas nesse departamento. Ademais, no Quadro 3, são apresentadas as variáveis da equação de tempo formuladas para o Almoxarifado.

Tabela 1: Custos apurados no Almoxarifado

\begin{tabular}{|l|r|}
\hline \multicolumn{1}{|c|}{ Recursos } & \multicolumn{1}{c|}{$\begin{array}{c}\text { Custos mensais (em } \\
\text { R\$) }\end{array}$} \\
\hline Funcionários & $4.097,56$ \\
\hline Depreciação & 985,23 \\
\hline Aluguel e Seguro & $3.591,58$ \\
\hline Material de Consumo & 278,54 \\
\hline Serviços gerais & $\mathbf{7 5 8 , 3 0}$ \\
\hline Total & $\mathbf{9 . 7 1 1 , 2 1}$ \\
\hline & 2 \\
\hline Número de funcionários (em unidades): & 16.896 \\
\hline $\begin{array}{l}\text { Minutos disponíveis (capacidade efetiva - em } \\
\text { minutos): }\end{array}$ & $\mathbf{0 , 5 7}$ \\
\hline Custo por minuto (em R\$): & \\
\hline
\end{tabular}


Tabela 2: Equações de tempo formuladas para o Almoxarifado

\begin{tabular}{|c|c|}
\hline Atividade & Equação de tempo \\
\hline Receber e estocar mercadoria (RE) & $R E=1 \times X_{1}+2 \times X_{2}+4 \times X_{3}+0,5 \times X_{4}$ \\
\hline Identificar e expedir mercadoria (IE) & $I E=2 \times X_{1}+4 \times X_{2}+1 \times X_{3}+0,5 \times X_{4}+5 \times X_{5}$ \\
\hline
\end{tabular}

\begin{tabular}{|c|c|}
\hline Atividade & Descrição da variável \\
\hline $\mathrm{RE}$ & $\begin{array}{c}\text { X1 } \rightarrow \text { Número de itens } \\
\text { X2 } \rightarrow \text { Estocagem no primeiro andar (caso se trate do primeiro andar, "1", do } \\
\text { contrário, "0") } \\
\text { X3 } \rightarrow \text { Estocagem no segundo andar (caso se trate do segundo andar, "1", do } \\
\text { contrário, "0") } \\
\text { X4 } \rightarrow \text { Número de itens distintos }\end{array}$ \\
\hline IE & 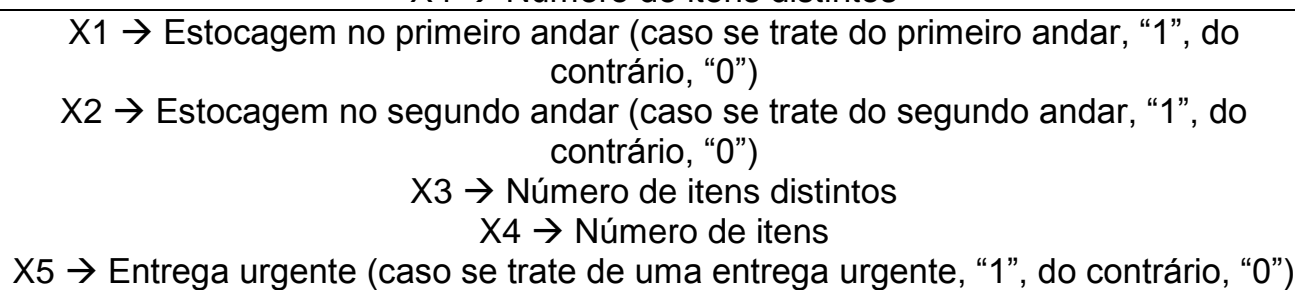 \\
\hline
\end{tabular}

Quadro 3: Descrição das variáveis das equações de tempo formuladas para o Almoxarifado

Para exemplificar, destacam-se os seguintes eventos para a equação de tempo da atividade IE desempenhada pelo Almoxarifado: (1) caso a mercadoria se encontre no primeiro andar do referido departamento (estoque principal), demora-se em média 2 minutos para localizá-la, do contrário, 4 minutos; (2) cada item distinto, ou seja, mercadorias com características diferentes demandam aproximadamente 1 minuto para seu recolhimento e para ser levado à área de expedição; (3) considera-se que cada item (unidade) demanda 0,5 minutos para ser embalado; e (4) caso se trate de uma entrega urgente, deve-se entrar em contato com prestadores de serviço de transporte da região (normalmente com a utilização de motos), que demanda cerca de 5 minutos. Assim, para uma venda que demande 3 mercadorias diferentes no primeiro andar, sendo no total 15 itens para entrega urgente, os funcionários do Almoxarifado gastarão 17,5 minutos, ou seja, $\mathrm{R} \$ 10,06$ para recolher e expedir a mercadoria.

Por sua vez, com base na coleta de dados realizada no Departamento Financeiro, foram identificadas e modeladas as seguintes atividades: "Pagar funcionários" (PF), "Pagar Tributos" (PT), "Emitir nota fiscal (saída de mercadoria) " 
(EN), "Inserir nota fiscal de entrada" (IN), e "Pagar contas diversas" (PC). Na Tabela 3, destacam-se os custos apurados mensalmente com o Departamento Financeiro; enquanto na Tabela 4 apresentam-se as equações de tempo definidas para as atividades por ele desenvolvidas. Além disso, no Quadro 4 são descritas as variáveis da equação de tempo formuladas para o Departamento.

Tabela 3: Custos apurados no Departamento Financeiro

\begin{tabular}{|c|c|}
\hline Recursos & $\begin{array}{c}\text { Custos mensais (em } \\
\boldsymbol{R \$})\end{array}$ \\
\hline Funcionários & $4.315,97$ \\
\hline Depreciação & 50,1 \\
\hline Aluguel e Seguro & 182,62 \\
\hline Material de Consumo & 278,54 \\
\hline Serviços gerais & 38,56 \\
\hline Total & $\mathbf{4 . 8 6 5 , 7 9}$ \\
\hline & 2 \\
\hline Número de funcionários (em unidades): & 16.896 \\
\hline Minutos disponíveis (capacidade efetiva - em \\
minutos):
\end{tabular}

Tabela 4: Equações de tempo formuladas para o Departamento Financeiro

\begin{tabular}{|c|c|}
\hline Atividade & Equação de tempo \\
\hline Pagar funcionários (PF) & $P F=5 \times X_{1}+3 X_{2}+3 X_{3}$ \\
\hline Pagar tributos (PT) & $P T=2+X_{1} \times 0,5$ \\
\hline Emitir nota fiscal (saída de mercadoria) & $E N=3+1,5 \times X_{1}$ \\
\hline (EN) & $I N=2+1,5 \times X_{1}+2 \times X_{2}+2$ \\
\hline Inserir nota fiscal de entrada (IN) & $P C=10 \times X_{1}+2+1+2 \times X_{2}+X_{3} \times\left(5+10+X_{4}\right)+1$ \\
\hline Pagar contas diversas (PC)
\end{tabular}




\begin{tabular}{|c|c|}
\hline Atividade & Descrição da variável \\
\hline PF & $\begin{array}{l}\text { X1 } \rightarrow \text { Número de funcionários da empresa } \\
\text { X2 } \rightarrow \text { Número de funcionários que recebem vale-transporte } \\
\text { X3 } \rightarrow \text { Número de funcionários que recebem vale-refeição }\end{array}$ \\
\hline PT & $\mathrm{X} 1 \rightarrow$ Número de campos da guia \\
\hline EN & $\mathrm{X} 1 \rightarrow$ Número de itens diferentes \\
\hline IN & $\begin{array}{c}\mathrm{X} 1 \rightarrow \text { Número de itens diferentes } \\
\text { X2 } \rightarrow \text { Mercadoria sofre substituição tributária (caso se trate de uma mercadoria na qual } \\
\text { há substituição tributária, "1"; do contrário, "0") }\end{array}$ \\
\hline PC & $\begin{array}{c}\text { X1 } \rightarrow \text { Mercadoria ou não (se mercadoria, "1", caso contrário, "0") } \\
\text { X2 } \rightarrow \text { Pagamento realizado em bancos da empresas ou não (se trata de um banco da } \\
\text { empresa, "1", caso contrário, "0") } \\
\text { X3 } \rightarrow \text { Pagamento realizado em outros bancos (se trata de outro banco, "1", caso } \\
\text { contrário, "0") } \\
\text { X4 } \rightarrow \text { Pagamento atrasado ou não (se o pagamento se referir a valores atrasados, "1", } \\
\text { caso contrário, "0") }\end{array}$ \\
\hline
\end{tabular}

Quadro 4: Descrição das variáveis das equações de tempo formuladas para o Departamento Financeiro

No intuito de exemplificar a equação de tempo da atividade IN executada pelo Departamento Financeiro consideraram-se os seguintes eventos: (1) as funcionárias responsáveis pela atividade gastam inicialmente 2 minutos para acessar o ERP da empresa; (2) cada item distinto, ou seja, mercadoria com características diferentes demanda 1,5 minutos para ser inserido no referido sistema; (3) caso se trate de uma mercadoria que demande substituição tributária pelo governo mineiro, gastam-se mais 2 minutos para inseri-la em uma planilha para controle especial; e (4) despendem-se mais 2 minutos para se arquivar a nota fiscal e enviá-la à Contabilidade. Desse modo, para uma nota fiscal que possua 6 itens distintos, sendo que 5 deles estejam sob o regime de substituição tributária, as funcionárias do departamento supracitado despenderão 27 minutos, ou seja, $R \$ 7,78$.

Com base na coleta de dados realizada no Departamento de Vendas, foi identificada e modelada a atividade "Vender Mercadorias" (VM). Na Tabela 5 são descritos os custos apurados mensalmente com o Departamento de Vendas; enquanto na Tabela 6 apresenta-se a equação de tempo definida para a atividade desenvolvida. Ademais, no Quadro 5, são apresentadas as variáveis da equação de tempo formulada para o Departamento de Vendas. 
Tabela 5: Custos apurados no Departamento de Vendas

\begin{tabular}{|c|c|}
\hline Recursos & $\begin{array}{c}\text { Custos mensais (em } \\
\mathbf{R \$} \text { ) }\end{array}$ \\
\hline Funcionários & $26.470,67$ \\
\hline Depreciação & 350,68 \\
\hline Aluguel e Seguro & 639,18 \\
\hline Material de Consumo & 557,09 \\
\hline Custos com Vendas & $1.241,60$ \\
\hline Serviços gerais & 269,90 \\
\hline Total & $\mathbf{2 9 . 5 2 9 , 1 2}$ \\
\hline & 4,00 \\
\hline Número de funcionários (em unidades): & $33.792,00$ \\
\hline Minutos disponíveis (capacidade efetiva - em \\
minutos): & $\mathbf{0 , 8 7}$ \\
\hline Custo por minuto (em $\mathrm{R} \$$ ): & \\
\hline
\end{tabular}

Tabela 6: Equações de tempo formuladas para o Departamento de Vendas

\begin{tabular}{|c|c|}
\hline Atividade & Equação de tempo \\
\hline $\begin{array}{c}\text { Vender mercadorias } \\
(\mathrm{VM})\end{array}$ & $X_{1} \times\left(2+1,5 \times X_{2}+3\right)+X_{3} \times\left(5 \times X_{4}+2 \times X_{2}+4+3\right)+X_{5} \times\left(5 \times X_{2}+3\right)+15 \times X_{6}$ \\
\hline
\end{tabular}

\begin{tabular}{|c|c|}
\hline Atividade & Descrição da variável \\
\hline & $\begin{array}{r}\text { X1 } \rightarrow \text { Venda por meio do portal da internet (caso se trate de uma venda realizada via } \\
\text { portal da internet, "1"; do contrário, "0"). } \\
\text { X2 } \rightarrow \text { Número de itens distintos }\end{array}$ \\
& $\begin{array}{r}\text { X3 } \rightarrow \text { Venda por telefone ou por e-mail (caso se trate de uma venda realizada via } \\
\text { telefone ou e-mail, "1"; do contrário, "0"). } \\
\text { X4 } \rightarrow \text { Venda por telefone (caso se trate de uma venda realizada via telefone, "1"; do } \\
\text { contrário, "0"). }\end{array}$ \\
& $\begin{array}{r}\text { X5 } \rightarrow \text { Venda realizada no balcão da loja (caso se trate de uma venda realizada no } \\
\text { balcão da loja, "1"; do contrário, "0"). } \\
\text { XM } \rightarrow \text { Cliente cadastrado ou não (caso se trate de um cliente não cadastrado, "1"; do } \\
\text { contrário, "0"). }\end{array}$ \\
\hline
\end{tabular}

Quadro 5: Descrição das variáveis da equação de tempo formulada para o Departamento de Vendas

A título de ilustração, descreve-se a equação de tempo da atividade VM do Departamento de Vendas, que reflete os seguintes eventos: (1) caso a venda seja realizada via Portal na Internet, demanda-se 2 minutos para acessar o referido portal on-line, 1,5 minutos para inserir corretamente cada item distinto e mais 3 minutos para verificar o interesse do cliente e formatar e imprimir a ordem de venda (OV); (2) caso a 
venda seja realizada por telefone, demanda-se 5 minutos para identificar e entender as necessidades específicas do cliente (no caso de e-mail, tal fase é desconsiderada), 2 minutos para inserir em uma planilha comercial cada item distinto, 4 minutos para enviar um e-mail ao cliente com o orçamento e 3 minutos para formatar e imprimir a OV; (3) caso a venda seja feita presencialmente na própria loja pelo comprador, demanda-se, aproximadamente, 5 minutos para discutir, identificar e solicitar a procura de cada mercadoria solicitada pelo cliente e 3 minutos para formatar e imprimir a OV; e (4) caso o cliente não seja cadastrado na empresa, são necessários 15 minutos para cadastrá-lo e efetivar a compra. Assim, uma venda de 25 itens distintos, realizada por telefone para um cliente não cadastrado implicaria o uso de 77 minutos dos funcionários do Departamento de Vendas, ou seja, $R \$ 67,29$.

Com base na coleta de dados realizada na Gerência da Empresa Beta, foram identificadas e modeladas as seguintes atividades: "Comprar materiais" (CM) e "Liberar crédito" (LC). Na Tabela 7, apresentam-se os custos apurados mensalmente na Gerência; enquanto na Tabela 8 apresentam-se as equações de tempo definidas para as atividades identificadas. Ademais, no Quadro 6 são apresentadas as variáveis da equação de tempo formuladas no que se relaciona à Gerência.

Tabela 7: Custos apurados da Gerência

\begin{tabular}{|c|c|}
\hline Recursos & $\begin{array}{c}\text { Custos mensais (em } \\
\boldsymbol{R \$})\end{array}$ \\
\hline Funcionários & $33.744,48$ \\
\hline Depreciação & 125,24 \\
\hline Aluguel e Seguro & 456,56 \\
\hline Material de Consumo & 278,54 \\
\hline Serviços gerais & 96,39 \\
\hline Total & $\mathbf{3 4 . 7 0 1 , 2 1}$ \\
\hline & 2 \\
\hline Número de funcionários (em unidades): & $16.896,00$ \\
\hline Minutos disponíveis (capacidade efetiva - em \\
minutos):
\end{tabular}


Tabela 8: Equações de tempo formuladas para a Gerência

\begin{tabular}{|c|c|}
\hline Atividade & Equação de tempo \\
\hline Comprar mercadorias (CM) & $C M=2,5 \times X_{1}$ \\
\hline Liberar crédito (LC) & $L C=3+2 \times X_{1}+10 \times X_{2}$ \\
\hline
\end{tabular}

\begin{tabular}{|c|c|}
\hline Atividade & Descrição da variável \\
\hline $\mathrm{CM}$ & $\mathrm{X} 1 \rightarrow$ Itens distintos \\
\hline LC & $\begin{array}{c}\text { X1 } \rightarrow \text { Número de clientes pesquisados. } \\
\text { X2 } \rightarrow \text { caso haja dúvidas se o cliente é um bom ou um mau pagador (se houver } \\
\text { dúvidas, "1"; do contrário, "0"). } \\
\text { X3 } \rightarrow \text { número de empresas na situação apresentada na variável X2 durante a } \\
\text { consulta. }\end{array}$ \\
\hline
\end{tabular}

Quadro 6: Descrição das variáveis das equações de tempo formuladas para a Gerência

Para exemplificar, a equação de tempo da atividade LC desempenhada pela Gerência destaca os seguintes eventos: (1) necessita-se, inicialmente, de 3 minutos para acessar o sistema on-line da entidade que presta informações sobre crédito de clientes (serviço contratado pela Empresa Beta); (2) cada cliente, cujas informações são procuradas, demandam 2 minutos de pesquisa; e (3) caso ainda houver dúvidas da credibilidade do cliente na concepção da gerência, necessitam-se de mais 10 minutos para procurar informações (via telefone) com outras empresas que transacionaram com a mesma (informação disponibilizada no sistema on-line supracitado) para cada cliente nessa situação. Desse modo, uma pesquisa sobre a credibilidade de um cliente, cujas informações do sistema on-line não evidenciem claramente sua probabilidade de insolvência, consome aproximadamente 15 minutos do tempo da Gerência, ou seja, $\mathrm{R} \$$ 30,81 .

\section{DISCUSSÃO DOS RESULTADOS}

Esta seção visa apresentar uma discussão dos resultados obtidos por meio da pesquisa empírica. Inicialmente, destaca-se o fato de a Empresa Beta apresentar um ambiente considerado ideal pela maioria dos autores, tais como Kaplan e Anderson (2007) e Everaert et al. (2008b), para a implementação do TDABC: ambiente 
operacional muito dinâmico, milhares de produtos, diversos processos padronizados, além de utilizar um ERP.

Observou-se a necessidade do desenvolvimento de apenas algumas equações de tempo, que foram suficientes para representar as principais atividades de cada departamento da empresa estudada. Salienta-se o fato de algumas dessas atividades bastante complexas terem sido modeladas sem maiores problemas, ratificando a teoria exposta por Kaplan e Anderson (2004) e alguns estudos empíricos, tais como Everaert et al. (2008b).

O ERP da empresa, por seu turno, foi pouco utilizado no que tange ao desenvolvimento das equações de tempo. Isso ocorreu devido à grande imperícia apresentada tanto pelos funcionários quanto pelos gestores (eles tinham sérias dificuldades em utilizar vários módulos e funcionalidades do ERP). Essa imperícia acarretou várias limitações ao desenvolvimento das equações, devido à falta de padronização de algumas atividades. Contudo, uma vez que essa limitação advém da imperícia dos usuários do ERP, tal constatação não parece ir de encontro ao indicado por Kaplan e Anderson (2007) e comprovado em vários estudos, como Pernot et al. (2007) e Varilla et al. (2007). Estes estudos estabelecem que a facilidade de integração entre o TDABC e os ERPs é uma das grandes vantagens do novo modelo, permitindo custos baixos de implementação e manutenção da metodologia de custeio. Todavia, destaca-se que o ERP foi fundamental para a coleta de dados referentes a custos por parte dos pesquisadores, tal como apresentado anteriormente. Ademais, deve-se ressaltar que o referido sistema é também essencial nos processos administrativos da empresa (principalmente no caso do Departamento Financeiro).

Ressalta-se, também, a substancial contribuição do MS-Excel para o desenvolvimento do modelo do TDABC da Empresa Beta. Primeiramente, as planilhas eletrônicas do referido sistema permitiram o adequado tratamento das informações de custos utilizadas no modelo. Posteriormente, foi possível modelar as informações das equações de tempo, a partir da inserção de valores para realizar referências às células ao longo dos cálculos. Esse procedimento foi adotado para que qualquer alteração em um parâmetro fosse plena e simultaneamente reconhecida no resultado da equação de 
tempo. Ademais, acrescentou-se uma seção nas planilhas em MS-Excel para preenchimento por parte do analista das diversas variáveis de cada equação (" $X n^{\prime}$ ). Próximo a essa seção, indicaram-se os sentidos de cada uma das variáveis e o que significa cada um dos valores demandados. Por fim, em uma planilha à parte, apurouse o custo por minuto de cada departamento responsável pela execução das atividades. As alterações dos parâmetros das equações de tempo permitem a visualização rápida do efeito do custo unitário de cada transação sob condições específicas (parâmetros diferenciados). Na Tabela 9 apresentam-se os custos de diferentes procedimentos na atividade VM realizada pelo Departamento de Vendas.

Tabela 9: Diferentes parâmetros para a atividade VM

\begin{tabular}{|c|c|c|c|c|c|c|}
\hline \multicolumn{6}{|c|}{ Valor das Variáveis } & \multirow{2}{*}{$\begin{array}{c}\text { Custo da transação (considerando o } \\
\text { custo por minuto de } R \$ 0,87 \text { ) }\end{array}$} \\
\hline $\mathbf{X 1}$ & $\mathrm{X} 2$ & X3 & X4 & $\mathrm{X5}$ & X6 & \\
\hline 1 & 5 & 0 & 0 & 0 & 1 & 23,93 \\
\hline 0 & 10 & 1 & 1 & 0 & 0 & 26,97 \\
\hline 0 & 2 & 0 & 0 & 1 & 0 & 8,70 \\
\hline 0 & 15 & 1 & 1 & 0 & 1 & 48,72 \\
\hline 1 & 8 & 0 & 0 & 0 & 0 & 14,79 \\
\hline 0 & 1 & 0 & 0 & 1 & 0 & 4,35 \\
\hline 1 & 20 & 0 & 0 & 0 & 1 & 43,50 \\
\hline
\end{tabular}

Verifica-se, pela análise da Tabela 9, que há inúmeras possibilidades para realizar a atividade VM, tornando-a bastante complexa, principalmente devido ao grande número de variáveis existentes. Isso permite a assimilação de uma série de peculiaridades, refletindo o custo de cada transação distinta. Destaca-se que isso seria bastante difícil de se realizar dentro da abordagem convencional do $A B C$, demandando um dicionário de atividades (lista de atividades) bastante extenso e de difícil aplicação.

Ainda com base na atividade VM, apurou-se o tempo demandando do Departamento de Vendas em determinado mês para o desempenho da referida atividade. Esses dados foram provenientes do ERP da empresa. Foram considerados, contudo, alguns pressupostos para a realização da análise, uma vez que ainda não há controles que forneçam tal informação na Empresa Beta: (i) metade dos clientes que compraram no período não tinha cadastro na empresa; e (ii) $10 \%$ (venda de balcão), $12 \%$ (venda por e-mail), $75 \%$ (venda por telefone) e portal na Internet. Essas 
estimativas foram realizadas pelos pesquisadores com base em sua experiência durante os meses de pesquisa.

Tabela 10: Estimativa de tempo demandado de trabalho do Departamento de Vendas

\begin{tabular}{|c|c|c|}
\hline Venda & $\begin{array}{c}\text { Tempo em } \\
\text { minutos }\end{array}$ & $\begin{array}{c}\text { Custos da transação (considerando o custo por } \\
\text { minuto de } \mathbf{R} \mathbf{\$ 0 , 8 7 )}\end{array}$ \\
\hline Balcão & $1.400,20$ & $1.223,56$ \\
\hline E-mail & $1.935,76$ & $1.691,56$ \\
\hline Telefone & $20.816,00$ & $18.190,05$ \\
\hline Portal na internet & 674,25 & 589,19 \\
\hline Total & $\mathbf{2 4 . 8 2 6 , 2 1}$ & $\mathbf{2 1 . 6 9 4 , 3 7}$ \\
\hline $\begin{array}{c}\text { Capacidade } \\
\text { disponível }\end{array}$ & $33.792,00$ & $29.529,12$ \\
\hline Diferença & $\mathbf{8 . 9 6 5 , 7 9}$ & $\mathbf{7 . 8 3 4 , 7 5}$ \\
\hline
\end{tabular}

Fonte: Elaborado pelos autores

As horas apresentadas pela diferença não podem ser entendidas como ociosidade completa, tendo em vista que algumas atividades não-estruturadas demandam, no mínimo, parte desse tempo. Baseando-se que essas atividades não parecem ser passíveis de modelar, não é possível estimar, com precisão, o número de horas ociosas do Departamento de Vendas, o que se constitui em uma limitação do modelo.

Destacam-se os vários benefícios identificados pela aplicação do modelo TDABC na Empresa Beta: (a) modelagem de atividades complexas, inerente a esse tipo de empresa; (b) pouco tempo para o desenvolvimento do modelo (se comparado ao $A B C$ convencional); (c) utilização de um sistema simples como o MS-Excel para modelagem das equações de tempo; (d) estimativa da capacidade efetiva de cada departamento da empresa e da capacidade disponível não utilizada (ociosa); e (e) estimativa dos custos das várias atividades e seus efeitos sobre as transações de diferentes mercadorias (melhoria da análise da lucratividade das mercadorias e das transações). Todas essas vantagens ratificam argumentos de Kaplan e Anderson (2004; 2007), além de resultados de vários estudos empíricos: Everaert et al. (2008b) Pernot et al. (2007) e Varilla et al. (2007). 
Contudo, algumas limitações também foram observadas pelos pesquisadores durante o período de estudo na Empresa Beta: (a) apesar de alguns autores destacarem a maior objetividade do TDABC em relação ao $A B C$ convencional, verificouse, ainda, um grau de subjetividade no que tange à nova abordagem (principalmente devido à necessidade de entrevistas e das estimativas realizadas pelos pesquisadores); (b) a falta de padronização de algumas atividades (tal como a montagem de painéis eletrônicos) impediu sua modelagem em termos de equações de tempo; (c) a falta da documentação interna à empresa no desenvolvimento de algumas atividades dificultou bastante a apuração de alguns direcionadores; e (d) a apuração do tempo ocioso das atividades não é totalmente adequada, devido à existência de variáveis aleatórias ou pouco padronizadas que não podem ser refletidas no modelo TDABC.

\section{CONCLUSÕES}

Verificou-se, pela fundamentação teórica que apóia este trabalho e por meio do estudo de caso realizado, que o TDABC realmente apresenta vantagens em relação ao $A B C$ convencional. Dentre essas vantagens identificadas podem ser citadas a facilidade em modelar atividades complexas por meio das equações de tempo e o curto tempo de desenvolvimento do modelo, o que representa normalmente um custo menor de implementação. Essas vantagens observadas corroboram os resultados de vários estudos empíricos e o apregoado por Kaplan e Anderson (2004; 2007).

Contudo, também foram identificadas algumas limitações da abordagem do TDABC no estudo de caso realizado. Primeiramente, os pesquisadores constataram um grau de subjetivismo durante as entrevistas com funcionários para mensuração do tempo de realização das atividades e para a identificação das variáveis que as afetam. Dessa forma, algumas críticas como as de Goldratt e Cox (1997) e Corbett Neto (1997) sob a subjetividade da abordagem do $A B C$ convencional parecem continuar, pelo menos em parte, válidas. Ademais, a falta de padronização de algumas atividades impede sua modelagem por meio das equações de tempo. Tal constatação ratifica os argumentos de Sherrat (2005) e os achados do estudo de Souza et al. (2010). Algumas 
limitações observadas no desenvolvimento do modelo, contudo, não são derivadas da abordagem do TDABC, mas, sim, de peculiaridades do caso estudado.

Salienta-se que uma das principais vantagens apresentadas por Kaplan e Anderson $(2004 ; 2007)$, no que se refere ao TDABC, está relacionada à fácil integração entre esse modelo e os ERPs das empresas. Tal fato, também já foi observado em vários estudos empíricos, tais como Pernot et al. (2007) e Varilla et al. (2007). Contudo, no estudo conduzido na Empresa Beta, tanto os funcionários quanto os gestores da referida empresa tinham problemas em trabalhar em alguns módulos e nas funcionalidades do ERP, o que dificultou a integração de informações do sistema para dar suporte ao TDABC.

Ademais, a falta de documentação (formalidade) de algumas atividades realizadas no interior dos departamentos dificultou bastante a mensuração de alguns direcionadores utilizados pelo modelo TDABC proposto. Muito provavelmente, essa falta de formalidade é, pelo menos em parte, inerente a empresas de pequeno porte, tal como a Empresa Beta. Assim, sugere-se, para atenuar tais limitações, uma maior padronização das atividades desempenhadas pelos departamentos da empresa e um maior treinamento dos funcionários no que tange ao uso de sistemas de informações tais como os ERPs, para que sejam plenamente úteis, tanto como suporte a um modelo TDABC, quanto nas próprias atividades rotineiras da empresa, aumentando possivelmente a produtividade.

Por fim, destacam-se algumas limitações da pesquisa descrita neste trabalho. Primeiramente, por se tratar de um estudo de caso único, não é possível generalizar os resultados encontrados em uma abordagem indutiva. Ademais, o fato de se tratar de uma empresa de varejo de pequeno porte provavelmente compromete a comparabilidade com resultados encontrados em empresas de maior porte ou de setores muitos distintos do varejista. Estudos futuros poderiam desenvolver pesquisas em outras empresas, além de abranger outros setores, a fim de corroborar ou não os resultados apresentados neste trabalho. 
Aplicação do time-driven ABC em uma empresa varejista Antônio Artur de Souza, Ewerton Alex Avelar, Terence Machado Boina, Neiva Andrade Caires

\section{REFERÊNCIAS}

ATKINSON, A. A. et al. (2008). Contabilidade gerencial. (2 ed.). São Paulo: Atlas. 812 p.

BLOCHER, E. J.; CHEN, K. H.; LIN, T. W. (2002). Cost Management: A strategic emphasis. (2 ed.). Mass.: McGraw-Hill//rwin. 1010 p.

BOISVERT, H. (1999). Contabilidade por atividades: práticas avançadas. São Paulo: Atlas. $93 \mathrm{p}$.

BRASIL. Lei Complementar № 123, de 14 de Dezembro De 2006. Disponível em: http://www.planalto.gov.br/ccivil_03/Leis/LCP/Lcp123.htm. Acesso em 01/julho/2009.

BRIMSON, J. A. (1996). Contabilidade por atividades: uma abordagem de custeio baseado em atividades. São Paulo: Atlas. 229 p.

BRUGGEMAN, W.; ANDERSON, S. R.; LEVANT, Y. (2005). Modeling Logistics Costs using Time-Driven ABC: A Case in a Distribution Company. Working Papers of Faculty of Economics and Business Administration, Ghent University, Belgium 05/332, Ghent University, Faculty of Economics and Business Administration.

BRYON, K. et al. Time-driven activity-based costing for supporting sustainability decisions in pig production. Disponível em: http://www.crrconference.org/downloads/lauwers.pdf. Acesso em 16/junho/2008.

CARDINAELS, E.; LABRO, E. (2008). On the Determinants of Measurement Error in Time-Driven Costing. Accounting Review, 83(3):735-56.

COOPER, R.; KAPLAN, R. S. (1991). The Design of Cost Management Systems: Text, Cases and Readings. New Jersey: Prentice-Hall.

CORBETT NETO, T. C. (1997). Contabilidade de Ganhos. São Paulo: Nobel.

DEMEERE, N.; STOUTHUYSENA, K.; ROODHOOFT, F. (2009). Time-driven activitybased costing in an outpatient clinic environment: Development, relevance and managerial impact. Health Policy.

EVERAERT, P.; BRUGGEMAN, W.; De CREUS, G. (2008a). Sanac Inc.: From ABC to time-driven $A B C$ - An instructional case. Journal of Accounting Education, 26:118-54.

EVERAERT, P. et al. Cost modeling in logistics using time-driven ABC. (2008b). Experiences from a wholesaler. International Journal of Physical Distribution \& Logistics Management, v. 38(3):172-9. 
GERI, N.; RONEN, B. (2005). Relevance lost: the rise and fall of activity-based costing. Human Systems Management, 24:133-144.

GOLAURT, R. L. (2000). Custeio baseado em atividade (ABC) aplicado em um serviço de radiologia em unidade hospitalar. Florianópolis, SC. (Dissertação de mestrado). Universidade Federal de Santa Catarina - UFSC, 140 p.

GOLDRATT, E. M.; COX, J. (1997). A meta: um processo de aprimoramento contínuo. (39 ed.). São Paulo: Educator. 395 p.

HANSEN, D. R.; MOWEN, M. M. (2006). Cost management: accounting and control. (5 ed.). Mason Ohio: Thomson/South-Western. 1004 p.

HIRSCH, M. L. (2000). Advanced management accounting. (2 ed.). London: Thomson Learning. $712 \mathrm{p}$.

KAPLAN, R. S.; COOPER, R. (1998). Custo e desempenho: administre seus custos para ser mais competitivo. São Paulo: Futura. $376 \mathrm{p}$.

KAPLAN, R. S.; ANDERSON, S. R. (2004). Time-driven activity-based costing. Harvard Business Review, 82(11):131.

(2007). Time-driven Activity-based Costing - A Simpler and more powerful path to higher profits. Harvard Business School Press, Massachusetts. $270 \mathrm{p}$.

MAJID, J. A.; SULAIMAN, M. (2008). Implementation of activity based costing in Malaysia: A case study of two companies. Asian Review of Accounting. 16(1):39-55.

MALHOTRA, N. K. (2003). Pesquisa de marketing. São Paulo: Bookman. 720 p.

MALMI, T. (1999). Activity-based costing difusion across organizations: an exploratory empirical analysis of Finnish firms. Accounting, Organizations and Society, 24:649-672.

PERNOT, E.; ROODHOOFT, F.; ABBEELE, V. D. A. (2007). Time-driven activity-based costing for inter-library services: a case study in a university. The Journal of Academic Librarianship, 33(5):551-6.

RADELLI, T.; DURAN, O. (2000). Metodologia ABC: implantação numa microempresa. Gestão \& Produção, 7(2):118-135.

SHERRATT, M. (2005). Harvard Business Review. Fev, 83(2):144. 
SILVA, I. S. T. (2003). Um estudo do custeio baseado em atividades (ABC) na apuração de custos ambientais. Porto Alegre, RS. (Dissertação de mestrado). Universidade Federal do Rio Grande do Sul - UFRGS, 173 p.

SOUZA, A. A. et al. (2010). Análise da aplicabilidade do time-driven activity-based costing em empresas de produção por encomenda. Revista Universo Contábil, 6(1):6784.

VARILLA, M.; SEPÄNNEN, M.; SUOMALA, P. (2007). Detailed cost modelling: a case study in warehouse logistics. International Journal of Physical Distribution \& Logistics Management, 37(3):184-200.

WEGMANN, G.; NOZILE, S. (2008). The activity-based costing method developments: state-of-the art and case study. ICFAI University Journal of Accounting Research, Forthcoming, p. 1-17.

YIN, R. K. (2005). Estudo de caso: planejamento e métodos. (3 ed.). Porto Alegre: Bookman. 212 p.

Data de Submissão: 23/07/2010

Data de Aceite 31/05/2012 